\title{
Some Properties of $A_{P}$ Weight Function
}

\author{
Santosh Ghimire \\ Department of Engineering Science and Humanities, Institute of Engineering \\ Pulchowk Campus, Tribhuvan University, Kathmandu, Nepal \\ Corresponding author: santoshghimire@ioe.edu.np
}

Received: June 20,2016Ｒevised: July 25, $2016 \quad$ Accepted: July 28, 2016

\begin{abstract}
In this paper, we briefly discuss the theory of weights and then define $A_{1}$ and $A_{p}$ weight functions. Finally we prove some of the properties of $A_{P}$ weight function.
\end{abstract}

Key words: $A_{1}$ weight function, Maximal functions, $A_{p}$ weight function.

\section{Introduction}

The theory of weights play an important role in various fields such as extrapolation theory, vector-valued inequalities and estimates for certain class of non linear differential equation. Moreover, they are very useful in the study of boundary value problems for Laplace's equation in Lipschitz domains. In 1970, Muckenhoupt characterized positive functions $\mathrm{w}$ for which the Hardy-Littlewood maximal operator $M$ maps $L^{\mathrm{p}}\left(\mathrm{R}^{\mathrm{n}}, \mathrm{w}(\mathrm{x}) \mathrm{dx}\right)$ to itself. Muckenhoupt's characterization actually gave the better understanding of theory of weighted inequalities which then led to the introduction of $A_{p}$ class and consequently the development of weighted inequalities.

\section{Definitions}

Definition: A locally integrable function on $\mathrm{R}^{\mathrm{n}}$ that takes values in the interval $(0, \infty)$ almost everywhere is called a weight. So by definition a weight function can be zero or infinity only on a set whose Lebesgue measure is zero.

We use the notation $w(E)=\int_{E} w(x) d x$ to denote the w-measure of the set $\mathrm{E}$ and we reserve the notation $\mathrm{L}^{\mathrm{p}}\left(\mathrm{R}^{\mathrm{n}}, \mathrm{w}\right)$ or $\mathrm{L}^{\mathrm{p}}(\mathrm{w})$ for the weighted $\mathrm{L}^{\mathrm{p}}$ spaces. We note that $\mathrm{w}(\mathrm{E})<\infty$ for all sets $\mathrm{E}$ contained in some ball since the weights are locally integrable functions.

Definition: The uncentered Hardy-Littlewood maximal operators on $\mathrm{R}^{\mathrm{n}}$ over balls $\mathrm{B}$ is defined as

$$
M(f)(x)=\sup _{x \in B} \operatorname{Avg}|f|=\sup _{x \in B} \frac{1}{|B|} \int_{B}|f(y)| d y .
$$

Similarly the uncentered Hardy-Littlewood maximal operators on $\mathrm{R}^{\mathrm{n}}$ over cubes $\mathrm{Q}$ is defined as 


$$
M_{c}(f)(x)=\sup _{x \in Q} \operatorname{Avg}|f|=\sup _{x \in Q} \frac{1}{|Q|} \int_{Q}|f(y)| d y .
$$

In each of the definition above, the suprema are taken over all balls $B$ and cubes $Q$ containing the point $\mathrm{x}$. H-L maximal functions are widely used in Harmonic Analysis. For the details about the H-L maximal operators, see [2].

Definition: A function $w(x) \geq 0$ is called an $A_{1}$ weight if there is a constant $C_{1}>0$ such that

$$
\mathrm{M}(\mathrm{w})(\mathrm{x}) \leq \mathrm{C}_{1} \mathrm{w}(\mathrm{x})
$$

where $\mathrm{M}(\mathrm{w})$ is uncentered Hardy-Littlewood Maximal function given by

$$
M(w)(x)=\sup _{x \in B} \frac{1}{|B|} \int_{B} w(t) d t .
$$

If $\mathrm{w}$ is an $\mathrm{A}_{1}$ weight, then the quantity (which is finite) given by

$$
[w]_{A_{1}}=\sup _{Q \text { cubesinR }}\left(\frac{1}{|Q|} \int_{Q}|w(t)| d t\right)\left\|w^{-1}\right\|_{L^{\infty}(Q)}
$$

is called the $\mathrm{A}_{1}$ characteristic constant of $\mathrm{w}$.

Definition: Let $1<\mathrm{p}<\infty$. A weight $\mathrm{w}$ is said to be of class $\mathrm{A}_{\mathrm{p}}$ if $[w]_{A_{p}}$ is finite where $[w]_{A_{p}}$ is defined as

$$
[w]_{A_{p}}=\sup _{Q \text { cubesin } R^{n}}\left(\frac{1}{|Q|} \int_{Q}|w(x)| d x\right)\left(\frac{1}{|Q|} \int_{Q}|w(x)|^{\frac{-1}{p-1}} d x\right)^{p-1} .
$$

We remark that in the above definition of $A_{1}$ and $A_{p}$ one can also use set of all balls in $R^{n}$ instead of all cubes in $\mathrm{R}^{\mathrm{n}}$.

\section{Properties}

Now we state and prove some of the properties of $A_{P}$ weight functions:

Property 1: Let $k$ be a nonnegative measurable function such that $k, k^{-1}$ are in $L^{\infty}\left(R^{n}\right)$. Then if $w$ is an $A_{P}$ for some $1 \leq p<\infty$, then $k w$ is also an $A_{P}$ weight function.

Proof: For kw to be an $A_{P}$ weight function, we must show (as described in definition) $[k w]_{A_{p}}<$ $\infty$, where $[k w]_{A_{p}}$ is given by;

$$
[k w]_{A_{p}}=\sup _{Q \text { cubesin } R^{n}}\left(\frac{1}{|Q|} \int_{Q}|k w(x)| d x\right)\left(\frac{1}{|Q|} \int_{Q}|k w(x)|^{\frac{-1}{p-1}} d x\right)^{p-1} .
$$

Note that $\mathrm{k}$ and $\mathrm{k}^{-1}$ are in $\mathrm{L}^{\infty}\left(\mathrm{R}^{\mathrm{n}}\right)$, so we have $\|k\|_{L^{\infty}}<\infty$ and $\left\|k^{-1}\right\|_{L^{\infty}}<\infty$. Using this together with nonnegativity of $\mathrm{k}$ and $\mathrm{k}^{-1}$, we have

$$
\begin{aligned}
\left(\frac{1}{|Q|} \int_{Q}|k w(x)| d x\right) & \left(\frac{1}{|Q|} \int_{Q}|k w(x)|^{\frac{-1}{p-1}} d x\right)^{p-1} \\
= & \left(\frac{1}{|Q|} \int_{Q}(k w)(x) d x\right)\left(\frac{1}{|Q|} \int_{Q}(k w(x))^{\frac{-1}{p-1}} d x\right)^{p-1}
\end{aligned}
$$




$$
\begin{aligned}
& \quad=\left(\frac{1}{|Q|} \int_{Q} k(x) w(x) d x\right)\left(\frac{1}{|Q|} \int_{Q}(k(x))^{\frac{-1}{p-1}} \cdot(w(x))^{\frac{-1}{p-1}} d x\right)^{p-1} \\
& \leq\left(\frac{1}{|Q|} \int_{Q}\|k\|_{L^{\infty}} w(x) d x\right)\left(\frac{1}{|Q|} \int_{Q}\left(|| k^{-1} \|_{L^{\infty}}\right)^{\frac{-1}{p-1}} \cdot(w(x))^{\frac{-1}{p-1}} d x\right)^{p-1} \\
& \leq\left.|| k\right|_{L^{\infty}}\left(\left.|| k^{-1}\right|_{L^{\infty}}\right)^{-1}\left(\frac{1}{|Q|} \int_{Q} w(x) d x\right)\left(\frac{1}{|Q|} \int_{Q}(w(x))^{\frac{-1}{p-1}} d x\right)^{p-1} \\
& \leq|| k||_{L^{\infty}}\left(\left.|| k^{-1}\right|_{L^{\infty}}\right)^{-1}[w]_{A_{p}}<\infty .
\end{aligned}
$$

Now taking supremum over all cubes $\mathrm{Q}$ in $\mathrm{R}^{\mathrm{n}}$, we get $[k w]_{A_{p}}<\infty$. This shows that kw is an $\mathrm{A}_{\mathrm{P}}$ weight.

Property 2: Suppose that $\mathrm{w}$ is in $\mathrm{A}_{\mathrm{p}}$ for some $p \in[1 . \infty]$ and $0<\delta<1$. Then $w^{\delta}$ belongs to $\mathrm{A}_{\mathrm{q}}$ where $\mathrm{q}=\delta \mathrm{p}+1-\delta$. Moreover, $\left[w^{\delta}\right]_{A_{p}} \leq[w]_{A_{p}}^{\delta}$.

Proof: We have

$$
\begin{aligned}
& I_{B}(w)=\left(\frac{1}{|B|} \int_{B} w^{\delta}\right)\left(\frac{1}{|B|} \int_{B} w^{\delta(1-p)} d x\right)^{\frac{1}{p-1}} \\
& \left(\frac{1}{|B|} \int_{B} w^{\delta}\right)\left(\frac{1}{|B|} \int_{B} w^{1-q} d x\right)^{\frac{\delta}{q-1}}
\end{aligned}
$$

Let us denote $f(q)=\left(\frac{1}{|Q|} \int_{Q} u^{q} d x\right)^{\frac{1}{q}}, u \geq 0, q>0$. Next we claim that $\mathrm{f}(\mathrm{q})$ is an increasing function of q. For this we note that by Holder's inequality,

$$
\int_{Q} u^{q} d x \leq\left(\frac{1}{|Q|} \int_{Q} u^{q k} d x\right)^{\frac{1}{k}}\left(\int_{Q} 1^{k^{\prime}} d x\right)^{\frac{1}{k^{\prime}}}, \forall k>1, \frac{1}{k}+\frac{1}{k^{\prime}}=1
$$

Let $\mathrm{q}_{1}>\mathrm{q}$. Set $k=\frac{q_{1}}{q}>1$. One has

$$
\int_{Q} u^{q} d x \leq\left(\int_{Q} u^{q_{1}} d x\right)^{\frac{q}{q_{1}}}|Q|^{1-\frac{q}{q_{1}}}
$$

Taking q-th root both sides, we have $f(q)=\left(\frac{1}{|Q|} \int_{Q} u^{q} d x\right)^{\frac{1}{q}} \leq\left(\frac{1}{|Q|} \int_{Q} u^{q_{1}} d x\right)^{\frac{1}{q_{1}}}=f\left(q_{1}\right), \forall q>q_{1}$. This proves the claim that $\mathrm{f}(\mathrm{q})$ is an increasing function of q. Using the claim we have,

$$
\begin{aligned}
\left(\frac{1}{|B|} \int_{B} w^{\delta}\right)^{\frac{1}{\delta}} & \leq \frac{1}{|B|} \int_{B} w, \forall 0<\delta \\
& <1
\end{aligned}
$$

From (1) and (2), we have, 


$$
I_{B}(w) \leq\left(\frac{1}{|B|} \int_{B} w\right)^{\delta}\left(\frac{1}{|B|} \int_{B} w^{(1-q)} d x\right)^{\frac{\delta}{q-1}} \leq[w]_{A_{p}}^{\delta} .
$$

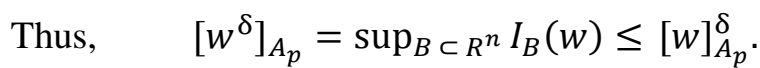

Property 3: Show that if the $A_{p}$ characteristics constants of a weight $w$ are uniformly bounded for all $\mathrm{p}>1$, then $\mathrm{w}$ is in $\mathrm{A}_{1}$.

Proof: As given, $[w]_{A_{p}}$ are uniformly bounded for all $\mathrm{p}>1$. Thus there exists a constant $\mathrm{M}<\infty$ such that $[w]_{A_{p}} \leq M$ for all $\mathrm{p}>1$. For a fixed $\mathrm{p}>1$, by definition we know that,

$$
[w]_{A_{p}}=\sup _{Q \text { balls in } R^{n}}\left(\frac{1}{|B|} \int_{B} w d x\right)\left(\frac{1}{|B|} \int_{B} w^{\frac{-1}{p-1}} d x\right)^{p-1} .
$$

So, $\left(\frac{1}{|B|} \int_{B} w d x\right)\left(\frac{1}{|B|} \int_{B} w^{\frac{-1}{p-1}} d x\right)^{p-1} \leq M$ for all balls B. Specifically, when $p \rightarrow 1$, the inequality holds. That is,

$$
\lim _{p \rightarrow 1^{+}}\left(\frac{1}{|B|} \int_{B} w(x) d x\right)\left(\frac{1}{|B|} \int_{B} w^{\frac{-1}{p-1}} d x\right)^{p-1}=\left(\frac{1}{|B|} \int_{B} w(x) d x\right)\left\|w^{-1}\right\|_{L^{\infty}(B)} \leq M .
$$

This is true because

$\lim _{p \rightarrow 1^{+}}\left(\frac{1}{|B|} \int_{B} w^{\frac{-1}{p-1}} d x\right)^{p-1}=\lim _{p \rightarrow 1^{+}}\left[\left.\left(\frac{1}{|B|}\right)^{p-1}|| w^{-1}\right|_{L^{\frac{1}{p-1}(B)}} ^{p-1}\right]=\lim _{p \rightarrow 1^{+}}|| w^{-1}||_{L^{\frac{1}{p-1}}(B)}^{p-1}$

and notice the fact that for $0<q<p \leq \infty,|| w^{-1}||_{L^{q}(B)} \leq|| w^{-1}||_{L^{p}(B)}$.

Now taking supremum over all balls B, we get,

$$
[w]_{A_{1}}=\left.\operatorname{Sup}_{B}\left(\frac{1}{|B|} \int_{B} w(x) d x\right)|| w^{-1}\right|_{L^{\infty}(B)} \leq M<\infty .
$$

Hence $w$ is in $A_{1}$ by definition.

There are other properties of $A_{p}$ which can be proved using the elementary analysis tools. For more properties, we refer $[1,2,3]$.

\section{Conclusion}

We studied $A_{1}$ and $A_{p}$ weight functions and we proved some important properties of these weight function using the elementary analysis tools.

\section{References}

[1] Ba nelos R and Moore CN (1991), Probabilistic Behavior of Harmonic Functions, Birkhauser Verlag, USA.

[2] Ghimire S (2014), Two Different Ways to Show a Function is an $A_{1}$ Weight Function, The Nepali Mathematical Sciences Report, 33(1): 53-58.

[3] Ghimire S (2014), Weighted Inequality, Journal of Institute of Engineering, 10(1): 121-124.

[4] Grafakos L (2009), Modern Fourier Analysis, Second Edition, Springer. 